\title{
Pulmonary Artery Aneurysm: A Very Rare Entity
}

Wan Irfan Wan Mustapha ${ }^{1}$, Ahmad Razali Md Ralib ${ }^{1}$, Norie Azilah Kamarudin ${ }^{2}$

${ }^{1}$ Department of Radiology, Kulliyyah of Medicine, International Islamic University of Malaysia

${ }^{2}$ Department of Radiology, Hospital Tengku Ampuan Afzan, Kuantan

Presenter: Wan Irfan Wan Mustapha

Pulmonary artery aneurysm (PAA) is defined as a focal dilatation of the pulmonary artery. It is noted to be a very rare entity. From literature reviews, only 8 cases of pulmonary artery aneurysms being identified from a total of 109,571 cases within an extended period of 100 years. This gives an incidence of about $0.0073 \%$. It is usually associated with some structural cardiac anomalies, structural vascular anomalies, pulmonary hypertension, vasculitis and infection. However idiopathic pulmonary artery aneurysm has also been identified in some cases. It can be classified as proximal or peripheral PAA depending on its location. Most of the patients are asymptomatic. The symptoms are only seen when complications occurred, such as bronchial or tracheal compression, dissection, rupture, thrombus formation causing pulmonary hypertension. It can be treated conservatively or surgically, especially in a symptomatic patient or if the size is more than $6 \mathrm{~cm}$. We reported a case of left pulmonary artery aneurysm, which is an incidental finding in a patient who underwent a CT pulmonary artery in view of a suspected pulmonary artery embolism when he presented with sudden onset of shortness of breath and left lower limb swelling and pain for one week. A left pulmonary artery aneurysm is seen measuring about $4.2 \mathrm{~cm} \mathrm{(W)} \times 3.9 \mathrm{~cm}(\mathrm{CC})$ extending to the proximal branch of descending pulmonary artery. Unfortunately, no further treatment can be given to the patient in view of the patient succumbed from sepsis shortly after the diagnosis. 